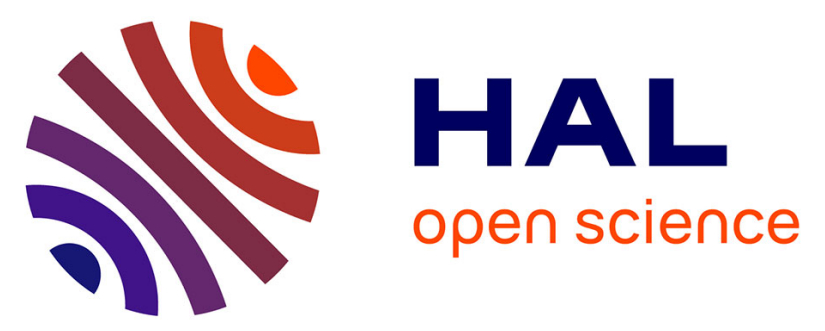

\title{
Normal serum ApoB48 and red cells vitamin E concentrations after supplementation in a novel compound heterozygous case of abetalipoproteinemia
}

M. Di Filippo, Sophie Collardeau-Frachon, A. Janin, S. Rajan, O. Marmontel, C. Decourt, A. Rubio, S. Nony, S. Dumont, C. Cuerq, et al.

\section{To cite this version:}

M. Di Filippo, Sophie Collardeau-Frachon, A. Janin, S. Rajan, O. Marmontel, et al.. Normal serum ApoB48 and red cells vitamin E concentrations after supplementation in a novel compound heterozygous case of abetalipoproteinemia. Atherosclerosis, 2019, 284, pp.75-82. 10.1016/j.atherosclerosis.2019.02.016 . hal-02195270

\author{
HAL Id: hal-02195270 \\ https://hal.science/hal-02195270
}

Submitted on 26 Jul 2021

HAL is a multi-disciplinary open access archive for the deposit and dissemination of scientific research documents, whether they are published or not. The documents may come from teaching and research institutions in France or abroad, or from public or private research centers.
L'archive ouverte pluridisciplinaire HAL, est destinée au dépôt et à la diffusion de documents scientifiques de niveau recherche, publiés ou non, émanant des établissements d'enseignement et de recherche français ou étrangers, des laboratoires publics ou privés. 


\title{
Normal serum ApoB48 and red cells vitamin E concentrations after supplementation in a novel compound heterozygous case of abetalipoproteinemia
}

\author{
Mathilde Di Filippo ${ }^{\mathrm{a}, \mathrm{b}, *}$, Sophie Collardeau Frachon ${ }^{\mathrm{b}, \mathrm{c}}$, Alexandre Janin ${ }^{\mathrm{a}, \mathrm{d}}$, Sujith Rajan ${ }^{\mathrm{e}}$ \\ Oriane Marmontel $^{\mathrm{a}, \mathrm{b}}$, Charlotte Decourt ${ }^{\mathrm{a}}$, Amandine Rubio ${ }^{\mathrm{f}, \mathrm{g}}$, Séverine Nony ${ }^{\mathrm{a}}$, Sabrina Dumont ${ }^{\mathrm{a}}$, \\ Charlotte Cuerq, ${ }^{\mathrm{b}, \mathrm{h}}$, Sybil Charrière ${ }^{\mathrm{b}, \mathrm{i}}$, Philippe Moulin ${ }^{\mathrm{b}, \mathrm{i}}$, Alain Lachaux ${ }^{\mathrm{j}}$, M. Mahmood Hussain ${ }^{\mathrm{e}}$, \\ Dominique Bozon ${ }^{\mathrm{a}}$, Noël Peretti ${ }^{\mathrm{b}, \mathrm{j}}$ \\ ${ }^{\mathrm{a}}$ Laboratoire de Biologie Médicale Multi Sites, Centre de Biologie et de Pathologie Est, Service de Biochimie et Biologie Moléculaire Grand Est, Hospices Civils de Lyon, Bron \\ cedex, F-69677, France \\ b INSERM U1060, Laboratoire Carmen, Université Lyon 1, INRA U1235, INSA de Lyon, CENS, Centre de Recherche en Nutrition Humaine Rhône Alpes, Villeurbanne F- \\ 69621, Oullins cedex, F-69921, France \\ ${ }^{\mathrm{c}}$ Laboratoire de Biologie Médicale Multi Sites, Centre de Biologie et de Pathologie Est, Institut de Pathologie, Hospices Civils de Lyon, Bron cedex, F-69677, France \\ ${ }^{\mathrm{d}}$ Université de Lyon, Université Claude Bernard Lyon 1, Institut NeuroMyoGène, CNRS UMR5310, INSERM U1217, Lyon, F-69622, France \\ ${ }^{\mathrm{e}}$ NYU Winthrop Hospital, 101 Mineola Blvd, Mineola, USA \\ ${ }^{\mathrm{f}}$ Gastroentérologie et Nutrition Pédiatrique Hôpital Couple Enfant, CHU de Grenoble Alpes, Grenoble, F-38043, France \\ ${ }^{\mathrm{g}}$ Laboratoire de Bioénergétique Fondamentale et Appliquée, INSERM U1055, Univ. Grenoble Alpes, F-38000, France \\ ${ }^{\mathrm{h}}$ Laboratoire de Biologie Médicale Multi Sites, Centre de Biologie et de Pathologie Sud, Service de Biochimie et Biologie Moléculaire, Hospices Civils de Lyon, Pierre, Benite \\ cedex, F-69495, France \\ ${ }^{\mathrm{i}}$ Fédération d'endocrinologie, maladies métaboliques, diabète et nutrition, Hôpital Louis Pradel, Hospices Civils de Lyon, Bron cedex, F-69677, France \\ ${ }^{\mathrm{j}}$ Service de Nutrition Pediatrique, Gastroenterologie and Hepatologie, Hôpital Femme Mère Enfants, Hospices Civils de Lyon, Bron cedex, F-69677, France
}

Background and aims: Abetalipoproteinemia (ABL) is a rare recessive monogenic disease due to MTTP (micro-somal triglyceride transfer protein) mutations leading to the absence of plasma apoB-containing lipoproteins. Here we characterize a new ABL case with usual clinical phenotype, hypocholesterolemia, hypotriglyceridemia but normal serum apolipoprotein B48 (apoB48) and red blood cell vitamin E concentrations.

Methods: Histology and MTP activity measurements were performed on intestinal biopsies. Mutations in MTTP were identified by Sanger sequencing, quantitative digital droplet and long-range PCR. Functional consequences of the variants were studied in vitro using a minigene splicing assay, measurement of MTP activity and apoB48

\footnotetext{
* Corresponding author. Hospices Civils de Lyon, Laboratoire de Biologie Médicale Multi Sites, Centre de Biologie et de Pathologie Est, Service de biochimie et biologie moléculaire Grand Est, Bron cedex, F-69677, France.

E-mail addresses: mathilde.di-filippo@chu-lyon.fr (M. Di Filippo), sophie.collardeau-frachon@chu-lyon.fr (S. Collardeau Frachon), alexandre.janin01@chu-lyon.fr (A. Janin), rajan.sujith@yahoo.co.uk (S. Rajan), oriane.marmontel@chu-lyon.fr (O. Marmontel), charlotte.dcrt@outlook.com (C. Decourt), arubio@chu-grenoble.fr (A. Rubio), severine.nony@chu-lyon.fr (S. Nony), sabrina.dumont@chu-lyon.fr (S. Dumont), charlotte.cuerq@chu-lyon.fr (C. Cuerq), sybil.charriere@chu-lyon.fr (S. Charrière), philippe.moulin@chu-lyon.fr (P. Moulin), alain.lachaux@chu-lyon.fr (A. Lachaux), Mahmood.Hussain@nyulangone.org (M.M. Hussain), bozon.dominique@orange.fr (D. Bozon), noel.peretti@chu-lyon.fr (N. Peretti).
} 
secretion.

Results: Intestinal steatosis and the absence of measurable lipid transfer activity in intestinal protein extract supported the diagnosis of ABL. A novel MTTP c.1868G > T variant inherited from the patient's father was identified. This variant gives rise to three mRNA transcripts: one normally spliced, found at a low frequency in intestinal biopsy, carrying the p.(Arg623Leu) missense variant, producing in vitro 65\% of normal MTP activity and apoB48 secretion, and two abnormally spliced transcripts resulting in a non-functional MTP protein. Digital droplet PCR and long-range sequencing revealed a previously described c.1067 +1217_1141del allele inherited from the mother, removing exon 10. Thus, the patient is compound heterozygous for two dysfunctional MTTP alleles. The p. (Arg623Leu) variant may maintain residual secretion of apoB48.

Conclusions: Complex cases of primary dyslipidemia require the use of a cascade of different methodologies to establish the diagnosis in patients with nonclassical biological phenotypes and provide better knowledge on the regulation of lipid metabolism.

\section{Introduction}

Abetalipoproteinemia (ABL; OMIM 200100) is a rare autosomal recessive disorder characterized by extremely low levels of plasma total cholesterol (TC) and triglycerides (TG) due to an almost complete absence of apolipoprotein B (apoB)-containing lipoprotein (apoB-LP) secretion [1]. This defect affects both apoB48-containing chylomicrons (CM) and apoB100-containing very-low density lipoproteins (VLDL) leading to an absence of low density lipoproteins (LDL) [2,3]. ABL is caused by a deficiency of the microsomal triglyceride transfer protein (MTP) large subunit encoded by MTTP, the microsomal triglyceride transfer protein gene [2-4]. The underlying mechanism involves the absence of MTP-protein disulfide isomerase (PDI) heterodimer that acts as a chaperone for the production of apoB-LP. This complex is required for the transfer of neutral lipids to nascent primordial apoB-LP and may facilitate their fusion with luminal lipid droplets [5,6]. In case of MTP deficiency, apoB cannot be lipidated properly and is degraded by the proteasome $[7,8]$.

ABL subjects are unable to secrete CM and VLDL from the intestine and liver, respectively. The absence of CM leads to lipid malabsorption, symptoms related to lipid-soluble vitamin and essential fatty acid deficiencies [9-11]. The absence of VLDL secretion induces liver steatosis leading to fibrosis and cirrhosis in many cases [11]. To date, at least 59 deleterious MTTP variants have been reported in ABL patients [11-17]. The molecular diagnosis is considered as the "gold-standard" diagnostic test, allowing the diagnosis of atypical ABL patients $[11,15,18]$ and establishing the differential diagnosis versus co-dominant familial compound heterozygous or homozygous hypobetalipoproteinemia (HoFHBL; OMIM 107730) due to deleterious variants on the $A P O B$ gene, encoding apoB ${ }^{11,19}$. Parents of ABL cases usually exhibit normal lipid profile whereas parents of Ho-FHBL cases exhibit mild hypobetalipoproteinemia $[19,20]$.

We report herein a new case of ABL occurring in childhood with typical clinical and lipid profile. However, the diagnosis was delayed by her normal plasma apoB48 concentration, mild hypolipoproteinemia in both parents, and the requirement of additional methods to Sanger sequencing allowing the subsequent identification of heterozygous copy number variations $(\mathrm{CNV})$.

\section{Materials and methods}

\subsection{Patient}

The patient is the first child of a non-consanguineous French family. She was delivered full-term after a normal pregnancy. Her birth measurements were normal (weight: $3.5 \mathrm{~kg}$, height: $50 \mathrm{~cm}$ ).

\subsection{Lipid profile, fat load, apoB electrophoresis, and vitamin $E$} determination

Lipid profile, CM, apoB48 and vitamin E concentration and apoB48 characterization can be found in Supplementary Material.

The proband received an oral fat load as described in Supplementary Material to assess the plasma TG concentration response.

\subsection{MTP activity on intestinal biopsy}

Intestinal biopsies of the proband and 11 control subjects without ABL were processed to obtain protein extracts and determine MTP activity as previously described $[18,21,22]$ and detailed in Supplementary Material. The results are expressed as UI per gram of total protein per hour.

\subsection{Variant and in silico analysis}

Following the extraction of genomic DNA (gDNA) from the blood, each of the coding exons, as well as the flanking intronic junctions of the MTTP, APOB, proprotein convertase subtilisin/kexin type 9 (PCSK9), and angiopoietin-like 3 (ANGPTL3) genes, was amplified by PCR and amplicons were sequenced as previously described [11]. Analysis of the mutations was performed using Alamut v2.11 as described in Supplementary Material.

\subsection{Protein activity and subcellular localization of the missense variant}

Three assays were performed as previously described [13,18,23] to evaluate the c.1868G $>\mathrm{T}$ p.(Arg623Leu) expression in COS cells: measurement of MTP activity, apoB48 secretion after cotransfection with the MTTP and P4HB (encoding PDI) cDNA, and subcellular localization after transfection with wild type (WT) or mutated MTTP cDNA C-terminally tagged with GFP (Supplementary Material).

2.6. Minigene splicing reporter assay and analysis of spliced products in HeLa cells after minigene transfection

As NM_000253.3(MTTP):c.1868G $>$ T variant affects the first base of exon 15 and may affect splicing, minigene splicing reporter assay was performed as previously described [18] and as detailed in Supplementary Material.

\subsection{RNA extraction from intestinal biopsy sample, PCR amplification and sequencing}

Total RNA was isolated, reverse transcribed into first strand cDNA; cDNA was then amplified and the PCR product was cloned into a TOPO vector and sequenced (Supplementary Material).

\subsection{Quantitative PCR and large rearrangement characterization}

Digital droplet quantitative PCR was performed from gDNA of the proband and her parents to detect CNV as detailed in Supplementary 
Material. Sequencing of the genomic deletion breakpoints was performed after long range PCR as previously described [11].

\section{Results}

\subsection{Patient (diagnosis and follow-up)}

Diarrhea appeared after the first week of life. She was referred to the hospital at 1 month of age for an acute pyelonephritis and at 4 months of age for growth retardation (weight: $3.8 \mathrm{~kg},<-2 \mathrm{SD}$, height: $58 \mathrm{~cm}$, $-2 \mathrm{SD}$, cranial perimeter: $37 \mathrm{~cm},-2 \mathrm{SD}$ ), with abdominal distension, diarrhea, and vomiting. Hepatomegaly was observed, blood transaminases were moderately elevated (Table 1 ) and hepatic steatosis was suspected because of the hyper-refringent liver observed at ultrasound examination. Her prothrombin ratio was decreased (18\%) due to vitamin K deficiency (normal factor V). A low-fat infant formula with a high medium-chain TG content (Monogen ${ }^{\oplus}$, Nutricia, Dublin, Ireland) was started. Liposoluble vitamin replacement was initiated with vitamin E (Vedrop "Orphan Europe Laboratories, Puteaux - France, 600 $\mathrm{U} / \mathrm{j}$ ), vitamin $\mathrm{K}$ (10 mg/week), vitamin A-313 (A313 ${ }^{\circ}$, Laboratoire Pharma Développement, Corbigny, France, 50000 U/week), vitamin D (Sterogyl ${ }^{\circ}$, Desma Pharma, Chiasso, Switzerland, 1600 U/day) in addition to omega 3 supplementation (Omacor ${ }^{\circ}$, Laboratoire Pierre Fabre Médicament, Ussel, France, 1 capsule containing EPA $460 \mathrm{mg}$ and DHA $380 \mathrm{mg}$, capsules were open and drank twice a week). The treatment stopped the diarrhea and improved growth.

At 14 months of age, her body weight was $9.1 \mathrm{~kg}$ (-1SD) and her height was $72 \mathrm{~cm}(-1 \mathrm{SD})$. She had no hepatomegaly, no diarrhea and no steatorrhea over a 3-day period strict low-fat diet (2-3 g/24 h of lipids). The liver elastometry was normal $(4.4 \mathrm{kPa}$ at 1 year of age) but the levels of blood transaminases remained moderately increased (Table 1). At 18 months of age, upper-endoscopy and duodenal biopsies showed vacuolated enterocytes (Fig. 1).

The patient is now 7 years of age and is in good health, without diarrhea under a strict low-fat diet. She presents no neurological, ophthalmological or cardiac abnormalities; a slight hepatic steatosis persisted upon ultrasound examination with normal liver elastometry $(3.3 \mathrm{kPa})$.

\subsection{Lipid profile, fat load, and apoB electrophoresis}

At diagnosis, the concentrations of $\mathrm{TC}$, $\mathrm{TG}$, apoB, and vitamin $\mathrm{E}$ were found to be dramatically low in the patient (Table 1 ) as were the concentrations of vitamin A $0.25 \mu \mathrm{mol} / \mathrm{L}$ (normal range, NR: 0.5-2.40), vitamin K $44 \mathrm{ng} / \mathrm{L}$ (NR: 100-1000) and beta-carotene $<0.10 \mu \mathrm{mol} / \mathrm{L}$ (NR: 0.30-0.89). After supplementation, serum vitamin E concentrations increased (Table 1) but remained under the NR whereas the red cells concentrations were found to be normal $(5.4 \mu \mathrm{mol} / \mathrm{L}$; NR: $2.0-7.8 \mu \mathrm{mol} / \mathrm{L}$ ) [24] as were the plasma and serum concentrations of vitamin A $(0.77 \mu \mathrm{mol} / \mathrm{L})$ and $\mathrm{K}(143 \mathrm{ng} / \mathrm{L}$ and $424 \mathrm{ng} / \mathrm{L}$ at 3 years of age). When the patient was diagnosed, the parents had serum LDL-C below normal concentration, which was more compatible with HoFHBL (dominant) than ABL (recessive); the serum LDL-C concentration of the parents was later found within normal range (Table 1).

When she was 1.5 years old, after an oral fat load (Table 1 ), the level of serum TG slightly increased from 0.05 to $0.10 \mathrm{mmol} / \mathrm{L}$. However, CM remained undetectable. Plasma $\mathrm{ApoB}$ electrophoresis revealed the presence of both apoB100 and apoB48 in the fasting state, and an increase of apoB48 and apoB100 after oral fat load (Fig. 2A and B), suggesting some intestinal lipid absorption and low amounts of intestinal and hepatic lipoprotein secretion.

ApoB48 concentration in fasting plasma at 3 different ages $(2,3.5$, and 4 years) was found to range from 8.40 to $11.06 \mathrm{mg} / \mathrm{L}$ in the normal IR (mean \pm 2 SD for controls: $5.81 \pm 6.18 \mathrm{mg} / \mathrm{L}, \mathrm{n}=11$ ). These values were higher than the limit of quantification $(0.25 \mathrm{mg} / \mathrm{L})$, and higher than apoB48 levels found in other ABL, in chylomicron retention disease (CMRD), and in Ho-FHBL patients (Fig. 2C).

\subsection{MTP activity on intestinal biopsy}

TG transfer activity in the intestinal biopsy at 18 months from the patient was not detectable $(<5 \%$ of the mean of controls subjects: mean \pm SD: $569 \pm 101 \mathrm{UI} / \mathrm{mg} / \mathrm{h}$, range: 393 to $761 \mathrm{UI} / \mathrm{mg} / \mathrm{h}$, $\mathrm{n}=11)$ whereas alkaline phosphatase activity (124 UI/g of protein) was normal (mean \pm SD for controls: $114 \pm 41 \mathrm{UI} / \mathrm{g}$, range: 56 to 173 UI/g of protein, $n=7$ ). These findings, showing a major MTP deficiency, were in agreement with ABL and not Ho-FHBL.

Table 1

Biological data for the patient and the parents.

\begin{tabular}{|c|c|c|c|c|c|c|c|c|c|c|c|}
\hline & Age & $\mathrm{TC}$ & TG & HDL-C & LDL-C & ApoA1 & ApoB & PL & vitamin $\mathrm{E}$ & ALAT & ASAT \\
\hline \multirow[t]{7}{*}{ Patient } & $4 \mathrm{~m}$ & 1.40 & 0.09 & 1.42 & ND & 1.07 & $<0.26$ & NA & 0.6 & 1.8 & 3.1 \\
\hline & $1.5 \mathrm{y}$ & 1.26 & 0.05 & 1.33 & ND & NA & $<0.04$ & 1.08 & $6.4^{\mathrm{b}}$ & NA & NA \\
\hline & $1.5 \mathrm{y} \mathrm{T}^{\mathrm{e}} \mathrm{h}^{\mathrm{e}}$ & 1.31 & 0.10 & 1.27 & ND & NA & $<0.04$ & 1.09 & NA & NA & NA \\
\hline & $1.5 \mathrm{y} \mathrm{T}^{\mathrm{e}} \mathrm{e}^{\mathrm{e}}$ & 1.22 & 0.07 & 1.28 & ND & NA & $<0.04$ & 1.09 & NA & NA & NA \\
\hline & 3 y $3 \mathrm{~m}$ & NA & NA & NA & NA & NA & NA & NA & $7.7^{\mathrm{c}}$ & 3.0 & 2.4 \\
\hline & $5 y$ & 1.22 & 0.05 & 1.21 & ND & NA & $<0.04$ & NA & $9.2^{\mathrm{d}}$ & NA & NA \\
\hline & $7 y$ & 1.24 & $<0.04$ & NA & NA & 0.94 & $<0.03$ & NA & $8.8^{\mathrm{d}}$ & 1.5 & 1.9 \\
\hline Sister & $3 \mathrm{~m}^{\mathrm{f}}$ & 3.63 & 2.42 & 1.41 & 1.12 & $\mathrm{NA}$ & 0.63 & NA & NA & NA & NA \\
\hline \multirow[t]{2}{*}{ Father } & 24 y $9 \mathrm{~m}$ & 2.30 & 1.33 & 0.65 & 1.04 & 1.35 & 0.50 & NA & NA & NA & NA \\
\hline & 24 y $11 \mathrm{~m}$ & 3.97 & 0.91 & 1.45 & 2.11 & NA & 0.58 & NA & NA & NA & NA \\
\hline
\end{tabular}

a. to ${ }^{\text {d. }}$ : under supplementation: ${ }^{\text {a. }}$.Vedrop ${ }^{\circ} 100 \mathrm{UI} / \mathrm{kg} / \mathrm{j}$, ${ }^{\mathrm{b}}$.Vedrop ${ }^{\circ} 120 \mathrm{UI} / \mathrm{kg} / \mathrm{j}$, ${ }^{\mathrm{c}}$.Cambridge ${ }^{\circ} 50 \mathrm{UI} / \mathrm{kg},{ }^{\mathrm{d}}$ Cambridge ${ }^{\circ} 100 \mathrm{mg} / \mathrm{kg}$, ${ }^{\mathrm{e}}$ numbers of hours after fat load, ${ }^{\mathrm{f}}$ after meal.

TC: total cholesterol; TG: triglycerides; HDL-C: High Density Lipoprotein cholesterol; LDL-C: low density lipoprotein cholesterol; Apo: apolipoprotein; PL: phospholipids; ALAT: alanine aminotransferase; ASAT: aspartate aminotransferase; ULN: upper limit of normal; m: month; y: year; NA: not available; ND: not detectable. Bold type: under normal range of the laboratory. Vitamin E normal range: 16 to $30 \mu \mathrm{mol} / \mathrm{L}$ 

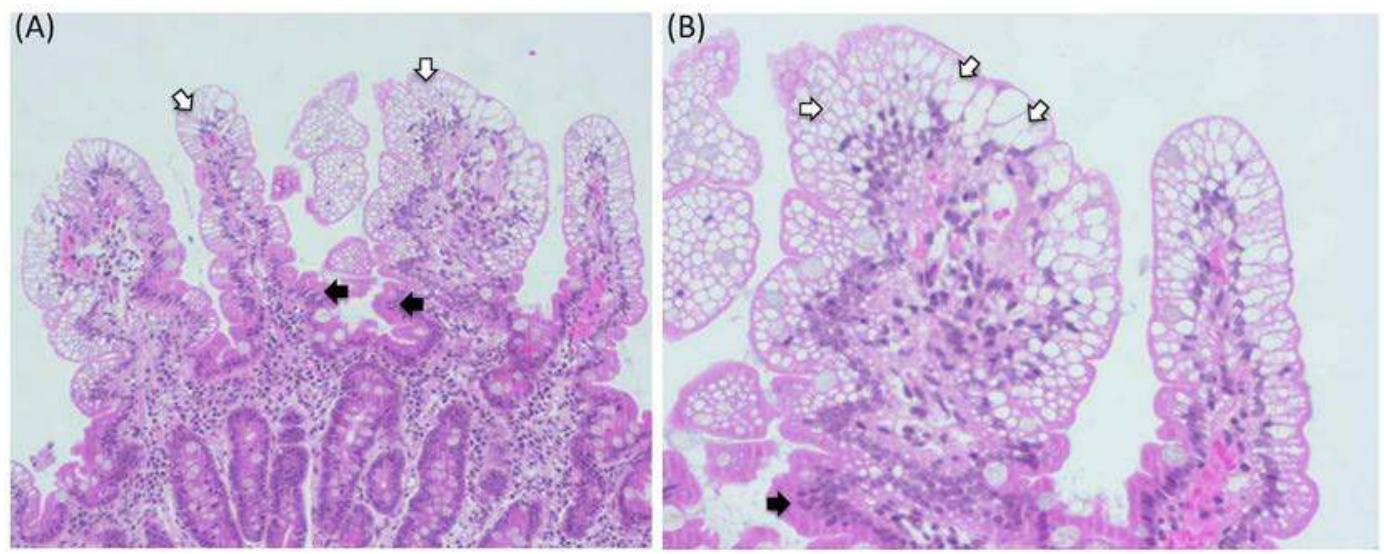

Fig. 1. Histological features in intestinal biopsy.

Duodenal biopsy obtained at 18 months and processed for Hematoxylin- Eosin- Saffron-staining. (A) $\times 100$ : intestinal villi mainly composed of vacuolated enterocytes and a few normal enterocytes as well as goblet cells. (B) x 200: focus on lipid droplets of variable sizes within the enterocytes. Black arrow: normal enterocytes; white arrows: vacuolated enterocytes.

A

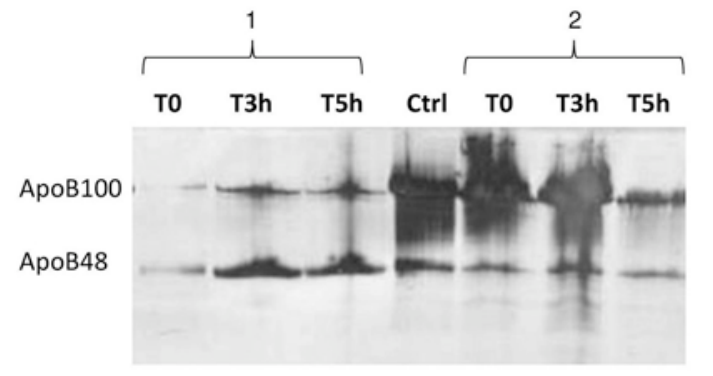

B

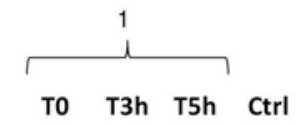

ApoB100

ApoB48

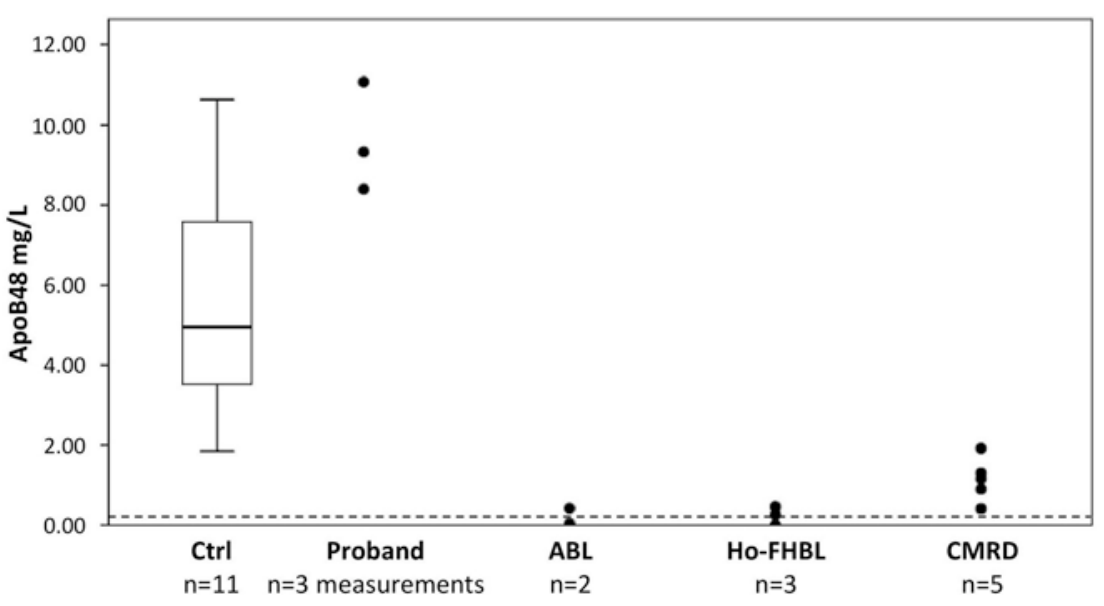

Fig. 2. ApoB electrophoresis and ApoB48 concentration.

(A and B) ApoB SDS-PAGE revealed by silver staining (A) or immunoblotting (B) before (T0) and after fat load for the proband (1), a normal subject (2); compared to a control (Ctrl: VLDL and chylomicrons). (C) Plasma apoB48 concentration in primary hypolipidemias and controls. Ctrl: controls; Proband $\mathrm{n}=3$ measurements (2, 3.5, and 4 years); ABL: abetalipoproteinemia; Ho-FHBL: homozygous or compound heterozygous hypobetalipoproteinemia; CMRD: chylomicron retention disease; dotted line: limit of quantification $(0.25 \mathrm{mg} / \mathrm{L})$.

\subsection{Identification of MTTP variants}

Two heterozygous, linked, nucleotide substitutions of the MTTP gene, both inherited from her father, were identified in the patient using Sanger sequencing. The first, NM_000253.3(MTTP): c.1868G $>$ T leads to p.(Arg623Leu) missense variant and affects the first base on exon 15. This variant is not described in the gnomAD database or in ClinVar. The second, NM_000253.3(MTTP): c.1981G > A, is a well described p.(Gly661Ser) missense variant. The frequency of this allele (rs113337987) is $2.6 \%$ in the gnomAD database. The patient had no deleterious variants in the other genes involved in familial hypocholesterolemia ( $A P O B, P C S K 9$, and ANGTL3). Her sister was not carrier of these single nucleotide variants on MTTP.

\subsection{Analysis of functional impacts of c.1868G > T, p.(Arg623Leu)}

\subsubsection{Impact on MTP activity and ApoB48 secretion}

The p.(Arg623Leu) variant affects a likely conserved residue. The substitution changes a basic hydrophilic to a hydrophobic residue. This missense variant is predicted by Polyphen to be "probably damaging" and by SIFT to be "tolerated".

COS-7 cells expressing MTTP c.582C > A (p.(Cys194*)) showed no measurable MTTP activity, but COS-7 cells expressing MTTP c.1868G > T (p.(Arg623Leu)) (the patient's variant) showed 60-65\% MTTP activity versus wild MTTP (Fig. 3A). The subcellular localization of the p.Leu623 missense mutant was then compared to the p.Arg623 (WT): both displayed a similar pattern, showing a reticular localization throughout the cytosol (Supplementary Fig. 1).

COS-7 cells expressing MTTP c.582C > A (p.(Cys194*)) variant 
A

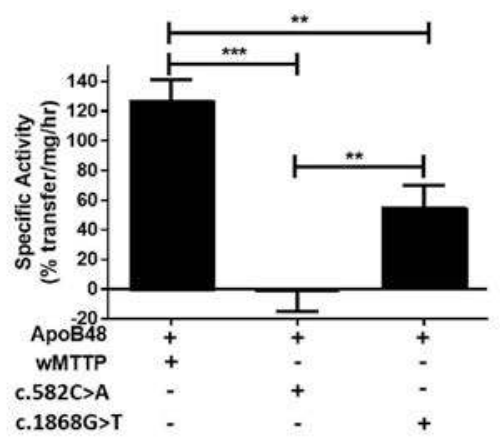

B

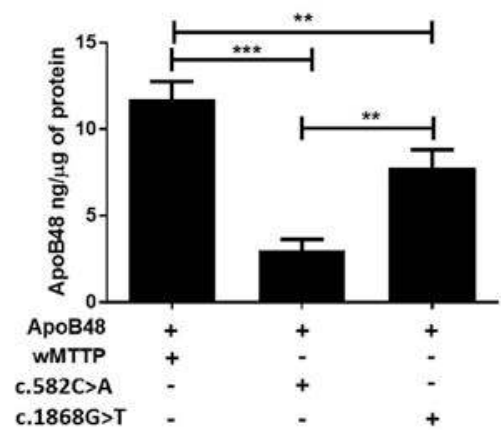

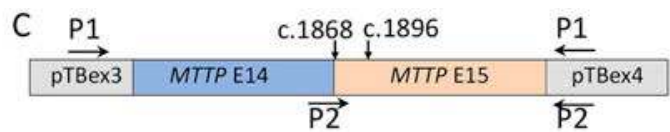

D

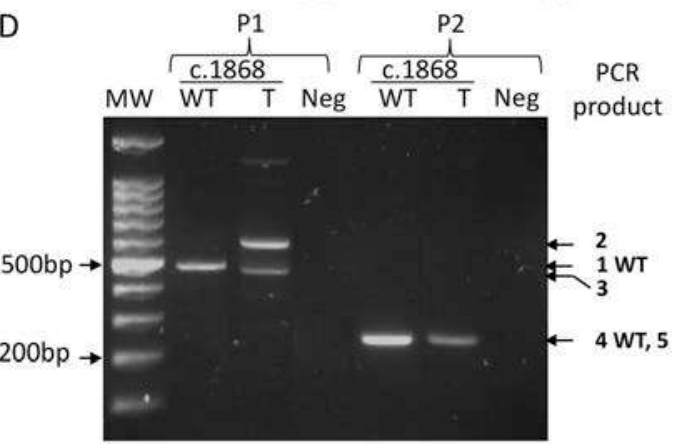

$\mathrm{E}$

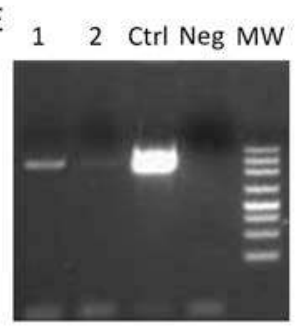

F

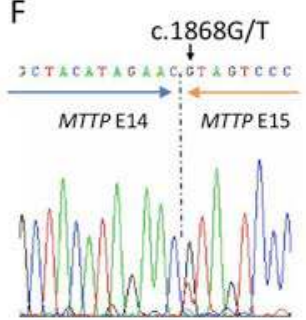

Fig. 3. In vitro and in vivo characterization of the c.1868G $>$ T, p.(Arg623Leu).

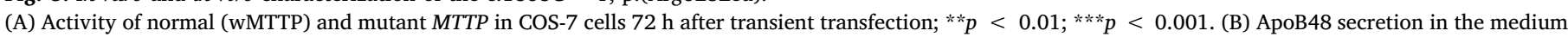

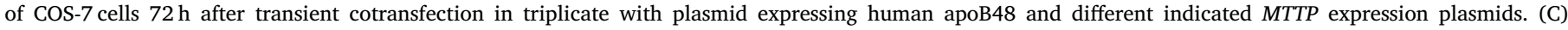

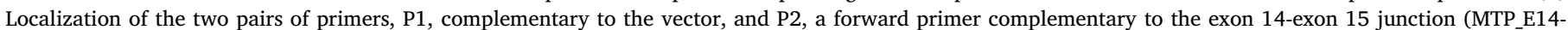

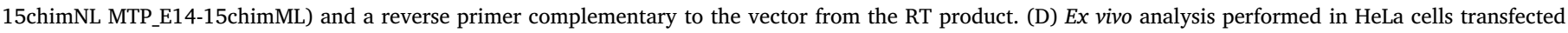

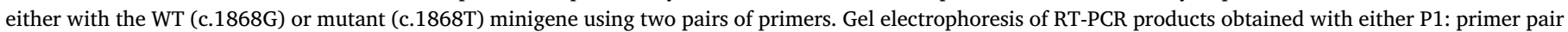

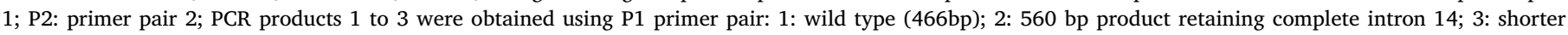

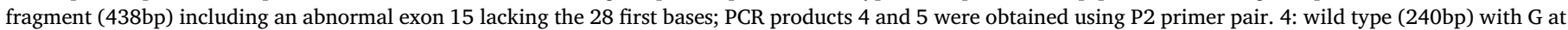

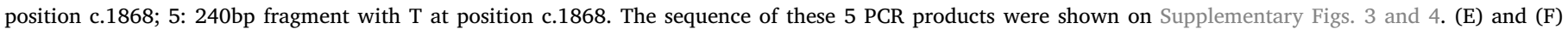

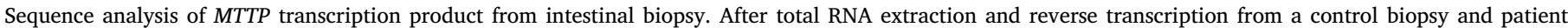

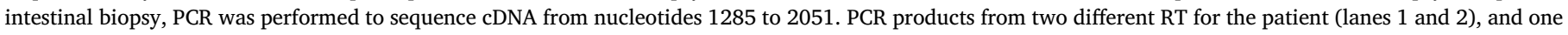
for the control biopsy (Ctrl) were analyzed by migration on agarose gel (E) and sequenced (F).

Neg: negative control; MW: molecular weight marker.

showed least apoB48 concentration in media, whereas COS-7 cells expressing MTTP c.1868G > T (p.(Arg623Leu)) (the patient's variant) showed $35-40 \%$ reduced apoB concentration in media compared to wild type (Fig. 3B). There was no significant difference in the level of protein expression of different MTTP mutants (Supplementary Fig. 2). These studies suggest that MTTP c.582C > A (p.(Cys194*)) variant does not transfer triglyceride and does not support apoB48 secretion. In contrast, MTTP c.1868G > T (p.(Arg623Leu)) (the patient's variant) is able to support triglyceride transfer and apoB48 secretion, albeit to a lower extent compared to WT MTTP.

\subsubsection{Impact of the $c .1868 G>T$ on splicing}

3.5.2.1. Splicing predictions. As this nucleotide change affects the first base of exon 15, all the tested algorithms predicted an effect on splicing with a decrease in the score for the acceptor site of intron 15 ranging from $-7.4 \%$ for SSF-like to a complete abolition for GeneSplicer, and NNSPLICE.

3.5.2.2. Minigene splicing reporter assay. To study the effect of this variant on splicing, RT-PCR products were analyzed using two pairs of primers (Fig. 3C).

A 466 bp expected RT-PCR fragment was produced after transfection of the WT minigene in HeLa cells (Fig. 3D, PCR product 1 and Supplementary Fig. 3, lanes 1, 2, and 3). In contrast, transfection of the mutant c.1868G $>\mathrm{T}$ minigene mainly produced 2 different RT-PCR fragments: a 560 bp product retaining complete intron 14, and a shorter fragment (438bp) including an abnormal exon 15 lacking the 28 first bases (Fig. 3D, PCR products 2 and 3 respectively, Supplementary Fig. 3, lanes 4, 5, 6, and Supplementary Fig. 4). Both transcripts would result in an aberrant protein with a premature stop codon. Minigene experiments indicated that the c.1868G $>\mathrm{T}$ leads mainly either to an activation of an exonic cryptic acceptor splice site at c.1896 in exon 15 or to a complete retention of intron 14. A very faint $466 \mathrm{bp}$ fragment, almost undetectable by gel electrophoresis, was also present in products from the mutant minigene.

To better characterize normally spliced products from the mutant minigene, cDNA was amplified with primer pair 2 (Supplementary Fig. 5). A PCR product of the expected size ( $240 \mathrm{bp}$ ) was obtained from RT PCR of both minigenes, WT and mutant, and Sanger sequencing confirmed the presence of the correct junction of exons 14 and 15 for both minigenes (with the presence of $\mathrm{G}$ and $\mathrm{T}$ at c.1868; Fig. 3D, PCR products 4 and 5, Supplementary Fig. 5). This result confirmed that a very small amount of a normally spliced transcript was also produced from the mutant minigene (Supplementary Fig. 6).

3.5.2.3. Amplification and sequence analysis of cDNA from intestinal biopsies. To study the in vivo effect of the c.1868G $>$ T variant, RTPCR of RNA prepared from the patient's biopsies was carried out spanning nucleotides 1285 to 2051 of MTTP cDNA. One PCR product exhibiting the expected size was found (Fig. 3E); this confirmed that the two misspliced mRNA transcripts from the variant containing the premature termination codons were targeted for nonsense-mediated 
decay and degraded. As expected, the sequence analysis of this transcript showed the presence of a normal sequence with a G/T mosaicism at positions c.1868 (Fig. 3F) and a G/A at c.1981 (from the paternal p.Gly661Ser variant).

\subsection{Detection and characterization of a copy number variation (CNV)}

As the patient exhibited a phenotype in agreement with ABL and only one heterozygous variant was found by Sanger sequencing, CNV were studied using digital droplet PCR. No quantitative difference was observed for exons $3,5,6,11,1317,18$, and 19 compared to the reference, while exon 10 of the proband and her mother exhibited approximately $50 \%$ of amplification products compared to the reference suggesting a heterozygous deletion (Fig. 4A).

After long-range PCR (Fig. 4B) and sequencing, the deletion breakpoints were identified (Fig. $4 \mathrm{C}$ and D): the patient, her mother and her sister presented a 2198bp deletion, which begins in the intron 9 and ends in exon 10, the NM_000253.3(MTTP):c.1067+1217_1141del variant, which was previously described as c.1068-2127_1138del. Deletion of the first 71 bases of exon 10 would induce a premature stop codon (p.(Pro357Phefs*4).

\section{Discussion}

We report the result of in-depth MTTP gene analysis in a proband with very low serum TG and TC but normal apoB48 concentration. The phenotype let to suggest either an ABL or Ho-FHBL diagnosis.

The phenotypic differential diagnosis between ABL and Ho-FHBL is often difficult as they can share similar clinical and biological manifestations [11]. The consideration of the lipid profile of the proband's parents is valuable: MTTP obligate heterozygotes usually have normal lipid profile while $A P O B$ heterozygotes are expected to have LDL-C and apoB levels below the 5th percentile according to age and sex $[11,19,25]$. Accordingly, mild hypocholesterolemia in both proband's parents was suggestive of Ho-FHBL in the proband. However, the

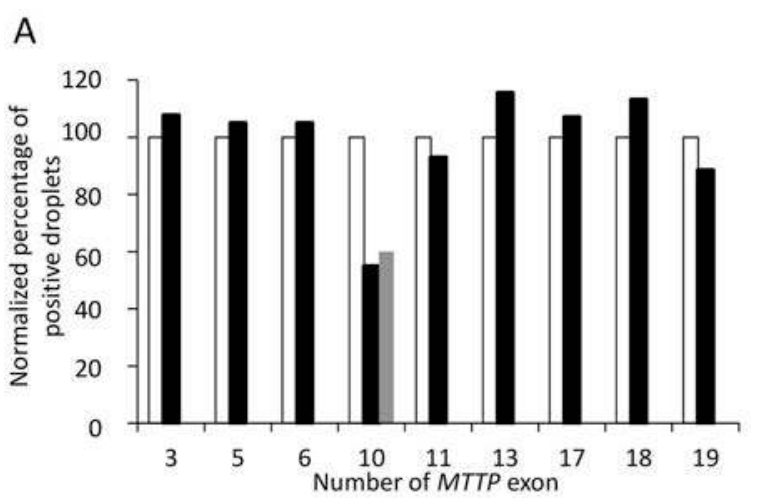

B

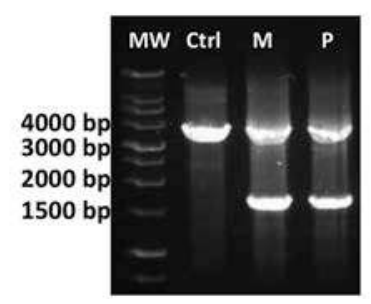

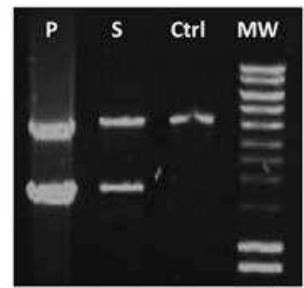

parents follow-up showed a fluctuant hypobetaliproteinemic lipid profile, and in the literature, $14 \%$ of the heterozygous carriers of deleterious MTTP variants were previously found to have low plasma LDL$\mathrm{C}$ and apoB [17]. In these cases, genetic analysis is mandatory to distinguish ABL from Ho-FHBL, and to consistently establish the diagnosis.

In the patient presented herein, intestinal biopsies were available, and the absence of detectable MTP activity suggested the diagnosis to ABL. However, Sanger sequencing detected only a new heterozygous variant of unknown significance, p.(Arg623Leu), on the MTTP paternal allele. The p.(Arg623Leu) is predicted to be surface exposed (Supplemental Fig. 7) in C-terminal ß-sheets domain of MTP mediating the lipid-binding and transfer catalytic activity of MTP [20]. Other missense variants in this domain and have been implicated in defective lipid transfer activity [11,26-28]. Since the impact of this missense variant was unclear and furthermore fasting concentration of apoB48 was normal and increasing after fat load, cellular functional tests were undertaken to decipher the functionality of this new variant. Its residual activity, correct subcellular localization and the remaining apoB48 secretion in the medium found in vitro strongly suggest that, unlike arginine at position 540 supposed to form a salt bridge with PDI [29], this residue at position 623 is less critical for MTP function. Additionally, in vitro, the c.1868T variant produced two major abnormally spliced transcripts and a very low level of a normally spliced transcript carrying the missense variant p.623Leu. Interestingly, ex vivo, in intestinal biopsy, only the normally spliced transcript carrying the missense variant was found, but at a very low level. This variant was consequently classified as pathogenic according to the American College of Medical Genetics and Association of Medical Pathologists (ACMG) guidelines [30].

Subsequent quantitative PCR allowed the identification of the maternal deleterious variant. This case underlines the necessity to routinely detect CNV in MTTP. Until now, CNV were reported in only 5 ABLpatients, for which the molecular mechanism (uniparental disomy, contiguous large gene deletion, or a homozygous deletion of an exon [11,31-34]) allowed detection by Sanger sequencing, whereas

C

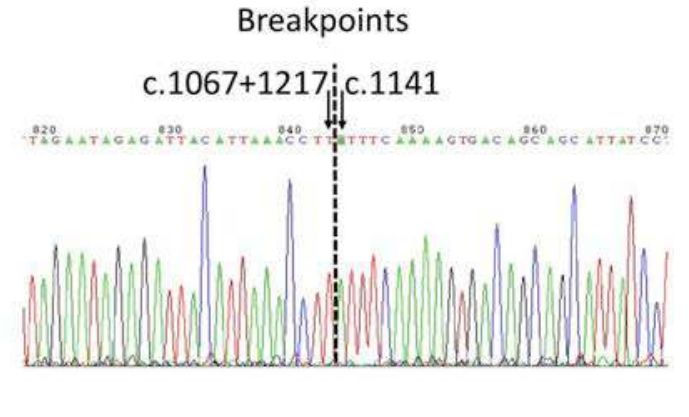

D

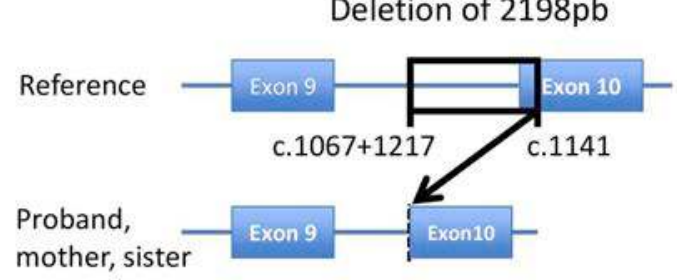

Fig. 4. Characterization of the c.1068-2127_c.1138del large genomic rearrangement.

(A) Digital droplet PCR: normalized percentage of positive droplets was represented for each tested exon. Deletion was suspected in case of percentage lower than $50 \%$. White bars: father, used as control; Black bars: proband; grey bars: mother. (B) Long range amplification from gDNA expected size for control 3876 bp; MW: molecular weight; Ctrl: control; M: mother; P: proband; S: sister. (C) Sequence of the deleted PCR product; the black line shows breakpoints. (D) Schematic representation of the deletion. 
heterozygous CNV are undetectable by Sanger sequencing. Consequently, it is of utmost importance to develop methods that allow the detection of CNV such as quantitative PCR or next-generation sequencing (NGS) for routine diagnosis [35-37], and therefore to allow the simultaneous detection of both single nucleotide variations and $\mathrm{CNV}$, as recently demonstrated in other dyslipidemias [38,39].

The same partial deletion of exon 10 has previously been described in another apparently unrelated homozygous patient [11]. The translation product of this variant is predicted to create a premature termination codon eliminating the lipid-binding cavity and the central region involved in the interaction with PDI [5,40,41]. The exon 10 deletion, caused by other molecular defects, leads to the production of a smaller MTTP protein, detectable but unable to interact with PDI, devoid of activity and shown to prevent the apoB secretion [4,29,42].

To the best of our knowledge this is the first report of normal plasma apoB48 levels associated with an increase of apoB after fat load in a clinically typical ABL case. ApoB is usually not detected in serum even after fat load in clinically typical ABL patients $[11,43,44]$. In the patient described herein, the normal apoB48 secretion could be explained by the residual activity of the heterozygous new missense MTTP variant. Although this residual activity was not measurable in the tested biopsy, the limited amount of tissue might have hampered the sensitivity of this ex vivo test.

The case presented is of particular interest because it has been previously established that sufficient apoB lipidation was conditional to avoid rapid apoB presecretory degradation [45]. In this patient, normal apoB48 concentration but very low apoB100 amount, undetectable using classical immunoturbidimetric measurement, was found as well as a raise in apoB plasma concentration after fat load whereas serum TG only increased to a maximum of $0.10 \mathrm{mmol} / \mathrm{L}$ and $\mathrm{CM}$ were not detectable. This observation raises the hypothesis of differential pathways in intestinal and hepatic cells regarding apoB proteasomal degradation and secretion of apoB. In accordance with the patient reported herein, in liver tissue-specific knockout $M t t p^{\Delta / \Delta}$ mice and in cultured murine primary hepatocytes treated with a MTP inhibitor, a partial inactivation of MTTP lowered apoB100 levels in plasma by 90 to $>95 \%$ whereas the secretion of apoB48 was only slightly decreased, indicating that secretion of apoB100 is much more sensitive to MTTP activity than apoB48 $[46,47]$. Despite the presence of detectable apoB48, this proband exhibited a complex profile of fat-soluble vitamin deficiency.

Vitamin $\mathrm{E}$ is thought to be incorporated into $\mathrm{CM}$ and transported by lipoproteins in serum. Despite the normal concentration of apoB48 in the patient presented herein, supporting secretion of apoB48-LP from the intestine, her vitamin E plasma concentrations were as low as those reported in other ABL patients in agreement with the very low level of its serum transporter, the LDL [11,13,20,29,42,48-54]. However, the normal concentration of vitamin $\mathrm{E}$ in red blood cells despite the absence of CM detected after fat load, supports additional mechanisms allowing the saving of vitamin $\mathrm{E}$ absorption in the context of vitamin $\mathrm{E}$ supplementation under a strict low-fat diet and poorly-lipidated apoB48 secretion. This might involve cooperation between regulation of vitamin E transport, HDL production and residual apoB48 secretion by enterocytes [55]. Additional studies will be needed to carefully characterize size and composition of the lipoproteins from the proband, when she will be adult due to the amount of plasma needed in abetalipoproteinemic patient.

In conclusion, we have shown that the main functional impairment of the c.1868G > T MTTP variant is a splicing defect that produces a small amount of normally spliced transcript enabling some residual MTP activity and apoB48 secretion. ABL patients with incomplete splicing defects may present with different clinical phenotype according to the molecular defect of the other allele. This case provides further insights into the genetic basis of the phenotypic diversity of ABL, regarding functional domains of MTTP, residual MTP activity, and their impact on apoB48 secretion and vitamin $\mathrm{E}$ absorption. It shows that methods allowing $\mathrm{CNV}$ detection are required to diagnose complex primary hypolipidemia, for which NGS may be of great interest.

\section{Conflicts of interest}

Philippe Moulin has received honoraria paid to his university for participating in clinical trials and/or advisory boards and/or symposia organized by: AKCEA, AMT, Chiesi, and Uniqure. The other authors have nothing to disclose.

\section{Author contributions}

Clinical assessments: AR NP.

Experiments: conception and design: MDF SCF AJ CC DB MH; performed the experiments: CD SN SD SR.

Analyzed the data: MDF SCF CD SN OM AJ DB CC MH PM.

Wrote the paper, approve the version to be published, agree to be accountable for all aspects of the work: all authors.

\section{Acknowledgments}

We would like to thank physicians and nurses of Pediatric Gastroenterology and Nutrition unit for carefully having taken the samples. We would like to warmly thank Dr Véronique Ducros for plasma vitamin E laboratory measurement and analytical performance specifications (Service de Biochimie, Centre Hospitalier Universitaire de Grenoble-Alpes, 38043 Grenoble, France). We would also like to give special thanks to Dr Agnes Sassolas (Hospices Civils de Lyon) for her previous work on the subject and Philip Robinson (DRCI, Hospices Civils de Lyon) for help in manuscript preparation.

\section{References}

[1] J.R. Burnett, A.J. Hooper, R.A. Hegele, Abetalipoproteinemia, in: M.P. Adam, H.H. Ardinger, R.A. Pagon, et al. (Eds.), GeneReviews((R)), Seattle (WA), 1993.

[2] C.C. Shoulders, D.J. Brett, J.D. Bayliss, et al., Abetalipoproteinemia is caused by defects of the gene encoding the $97 \mathrm{kDa}$ subunit of a microsomal triglyceride transfer protein, Hum. Mol. Genet. 2 (1993) 2109-2116.

[3] D. Sharp, L. Blinderman, K.A. Combs, et al., Cloning and gene defects in microsomal triglyceride transfer protein associated with abetalipoproteinaemia, Nature 365 (1993) 65-69.

[4] J.R. Wetterau, L.P. Aggerbeck, M.E. Bouma, et al., Absence of microsomal triglyceride transfer protein in individuals with abetalipoproteinemia, Science 258 (1992) 999-1001.

[5] M.M. Hussain, J. Shi, P. Dreizen, Microsomal triglyceride transfer protein and its role in apoB-lipoprotein assembly, J. Lipid Res. 44 (2003) 22-32.

[6] A. Sirwi, M.M. Hussain, Lipid transfer proteins in the assembly of apoB-containing lipoproteins, J. Lipid Res. 59 (2018) 1094-1102.

[7] R.A. Davis, R.N. Thrift, C.C. Wu, et al., Apolipoprotein B is both integrated into and translocated across the endoplasmic reticulum membrane. Evidence for two functionally distinct pools, J. Biol. Chem. 265 (1990) 10005-10011.

[8] M. Zhou, E.A. Fisher, H.N. Ginsberg, Regulated Co-translational ubiquitination of apolipoprotein B100. A new paradigm for proteasomal degradation of a secretory protein, J. Biol. Chem. 273 (1998) 24649-24653.

[9] G. Delpre, U. Kadish, I. Glantz, et al., Endoscopic assessment in abetalipoproteinemia (Bassen-Kornzweig-syndrome), Endoscopy 10 (1978) 59-62.

[10] N. Berriot-Varoqueaux, L.P. Aggerbeck, M. Samson-Bouma, et al., The role of the microsomal triglygeride transfer protein in abetalipoproteinemia, Annu. Rev. Nutr. $20(2000)$ 663-697.

[11] M. Di Filippo, P. Moulin, P. Roy, et al., Homozygous MTTP and APOB mutations may lead to hepatic steatosis and fibrosis despite metabolic differences in congenital hypocholesterolemia, J. Hepatol. 61 (2014) 891-902.

[12] S.A. Miller, J.R. Burnett, M.A. Leonis, et al., Novel missense MTTP gene mutations causing abetalipoproteinemia, Biochim. Biophys. Acta 1842 (2014) 1548-1554.

[13] M.T. Walsh, J. Iqbal, J. Josekutty, et al., Novel abetalipoproteinemia missense mutation highlights the importance of the N-terminal beta-barrel in microsomal triglyceride transfer protein function, Circ Cardiovasc Genet 8 (2015) 677-687.

[14] M. Gunduz, E. Ozaydin, M.B. Atar, et al., Microsomal triglyceride transfer protein gene mutations in Turkish children: a novel mutation and clinical follow up, Indian J. Gastroenterol. : Offc. J. Indian Soc. Gastroenterol. 35 (2016) 236-241. 
[15] M. Paquette, R. Dufour, R.A. Hegele, et al., A tale of 2 cousins: an atypical and a typical case of abetalipoproteinemia, J Clin Lipidol 10 (2016) 1030-1034.

[16] Y. Liu, D.M. Conlon, X. Bi, et al., Lack of MTTP activity in pluripotent stem cellderived hepatocytes and cardiomyocytes abolishes apoB secretion and increases cell stress, Cell Rep. 19 (2017) 1456-1466.

[17] M. Di Filippo, M. Varret, V. Boehm, et al., Post-prandial lipid absorption in seven heterozygous carriers of deleterious variants of MTTP in two abetalipoproteinemic families, J Clin Lipidol 13 (1) (2019 Jan - Feb) 201-212, https://doi.org/10.1016/j. jacl.2018.10.003 Epub 2018 Oct 24, PMID: 30522860.

[18] M. Di Filippo, H. Crehalet, M.E. Samson-Bouma, et al., Molecular and functional analysis of two new MTTP gene mutations in an atypical case of abetalipoproteinemia, J. Lipid Res. 53 (3) (2012 Mar) 548-555, https://doi.org/10.1194/jlr. M020024 Epub 2012 Jan 11, PMID 22236406.

[19] J. Lee, R.A. Hegele, Abetalipoproteinemia and homozygous hypobetalipoproteinemia: a framework for diagnosis and management, J. Inherit. Metab. Dis. 37 (2014) 333-339.

[20] R. Zamel, R. Khan, R.L. Pollex, et al., Abetalipoproteinemia: two case reports and literature review, Orphanet J. Rare Dis. 3 (2008) 19.

[21] H. Athar, J. Iqbal, X.C. Jiang, et al., A simple, rapid, and sensitive fluorescence assay for microsomal triglyceride transfer protein, J. Lipid Res. 45 (2004) 764-772.

[22] P. Rava, H. Athar, C. Johnson, et al., Transfer of cholesteryl esters and phospholipids as well as net deposition by microsomal triglyceride transfer protein, J. Lipid Res. 46 (2005) 1779-1785.

[23] M.T. Walsh, E. Di Leo, I. Okur, et al., Structure-function analyses of microsomal triglyceride transfer protein missense mutations in abetalipoproteinemia and hypobetalipoproteinemia subjects, Biochim. Biophys. Acta 1861 (2016) 1623-1633.

[24] C. Cuerq, L. Restier, J. Drai, et al., Establishment of reference values of alpha-tocopherol in plasma, red blood cells and adipose tissue in healthy children to improve the management of chylomicron retention disease, a rare genetic hypocholesterolemia, Orphanet J. Rare Dis. 11 (2016) 114.

[25] A.J. Hooper, J.R. Burnett, G.F. Watts, Contemporary aspects of the biology and therapeutic regulation of the microsomal triglyceride transfer protein, Circ. Res. 116 (2015) 193-205.

[26] R.A. Hegele, A. Angel, Arrest of neuropathy and myopathy in abetalipoproteinemia with high-dose vitamin E therapy, Can. Med. Assoc. J. 132 (1985) 41-44.

[27] Y. Wang, K. Tran, Z. Yao, The activity of microsomal triglyceride transfer protein is essential for accumulation of triglyceride within microsomes in McA-RH7777 cells. A unified model for the assembly of very low density lipoproteins, J. Biol. Chem. 274 (1999) 27793-27800.

[28] I. Khatun, M.T. Walsh, M.M. Hussain, Loss of both phospholipid and triglyceride transfer activities of microsomal triglyceride transfer protein in abetalipoproteinemia, J. Lipid Res. 54 (2013) 1541-1549.

[29] E.F. Rehberg, M.E. Samson-Bouma, B. Kienzle, et al., A novel abetalipoproteinemia genotype. Identification of a missense mutation in the $97-\mathrm{kDa}$ subunit of the microsomal triglyceride transfer protein that prevents complex formation with protein disulfide isomerase, J. Biol. Chem. 271 (1996) 29945-29952.

[30] S. Richards, N. Aziz, S. Bale, et al., Standards and guidelines for the interpretation of sequence variants: a joint consensus recommendation of the American College of medical genetics and genomics and the association for molecular pathology, Genet. Med. 17 (2015) 405-424.

31] X.P. Yang, A. Inazu, K. Yagi, et al., Abetalipoproteinemia caused by maternal isodisomy of chromosome $4 \mathrm{q}$ containing an intron 9 splice acceptor mutation in the microsomal triglyceride transfer protein gene, Arterioscler. Thromb. Vasc. Biol. 19 (1999) 1950-1955.

[32] L. Benayoun, E. Granot, L. Rizel, et al., Abetalipoproteinemia in Israel: evidence for a founder mutation in the Ashkenazi Jewish population and a contiguous gene deletion in an Arab patient, Mol. Genet. Metabol. 90 (2007) 453-457.

[33] D. Kotzot, Complex and segmental uniparental disomy updated, J. Med. Genet. 45 (2008) 545-556.

[34] A. Aminoff, E. Gunnar, M. Barbaro, et al., Novel mutations in microsomal triglyceride transfer protein including maternal uniparental disomy in two patients with abetalipoproteinemia, Clin. Genet. 82 (2) (2012 Aug) 197-200, https://doi.org/10 1111/j.1399-0004.2011.01828.x Epub 2011 Dec 29. No abstract available. Erratum in: Clin Genet. 2012 Aug;82(2):204.PMID: 22150066.

[35] S. Ceulemans, K. van der Ven, J. Del-Favero, Targeted screening and validation of copy number variations, Methods Mol. Biol. 838 (2012) 311-328, https://doi.org/ 10.1007/978-1-61779-507-7 15 PMID: 22228019.

[36] B.E. Slatko, A.F. Gardner, F.M. Ausubel, Overview of next-generation sequencing technologies, Curr Protoc Mol Biol 122 (2018) e59.

[37] P.L. Quan, M. Sauzade, E. Brouzes, dPCR: a technology review, Sensors 18 (2018) E1271.

[38] M.A. Iacocca, J. Wang, J.S. Dron, et al., Use of next-generation sequencing to detect LDLR gene copy number variation in familial hypercholesterolemia, J. Lipid Res. 58 (2017) 2202-2209.

[39] O. Marmontel, S. Charriere, T. Simonet, et al., Single, short in-del, and copy number variations detection in monogenic dyslipidemia using an NGS strategy, Clin. Genet (2018).

[40] C.J. Mann, T.A. Anderson, J. Read, et al., The structure of vitellogenin provides a molecular model for the assembly and secretion of atherogenic lipoproteins, J. Mol. Biol. 285 (1999) 391-408.

[41] J. Read, T.A. Anderson, P.J. Ritchie, et al., A mechanism of membrane neutral lipid acquisition by the microsomal triglyceride transfer protein, J. Biol. Chem. 275 (2000) 30372-30377.

[42] V. Pons, C. Rolland, M. Nauze, et al., A severe form of abetalipoproteinemia caused by new splicing mutations of microsomal triglyceride transfer protein (MTTP), Hum. Mutat. 32 (2011) 751-759.

[43] D.R. Illingworth, W.E. Connor, R.G. Miller, Abetalipoproteinemia. Report of two cases and review of therapy, Arch. Neurol. 37 (1980) 659-662.

[44] L. Magnolo, M. Najah, T. Fancello, et al., Novel mutations in SAR1B and MTTP genes in Tunisian children with chylomicron retention disease and abetalipoproteinemia, Gene 512 (2013) 28-34.

[45] J. Boren, M. Wettesten, A. Sjoberg, et al., The assembly and secretion of apoB 100 containing lipoproteins in Hep G2 cells. Evidence for different sites for protein synthesis and lipoprotein assembly, J. Biol. Chem. 265 (1990) 10556-10564.

[46] M. Raabe, M.M. Veniant, M.A. Sullivan, et al., Analysis of the role of microsomal triglyceride transfer protein in the liver of tissue-specific knockout mice, J. Clin. Investig. 103 (1999) 1287-1298.

[47] A. Kulinski, S. Rustaeus, J.E. Vance, Microsomal triacylglycerol transfer protein is required for lumenal accretion of triacylglycerol not associated with $\mathrm{ApoB}$, as wel as for ApoB lipidation, J. Biol. Chem. 277 (2002) 31516-31525.

[48] T.M. Narcisi, C.C. Shoulders, S.A. Chester, et al., Mutations of the microsomal triglyceride-transfer-protein gene in abetalipoproteinemia, Am. J. Hum. Genet. 57 (1995) 1298-1310.

[49] K. Ohashi, S. Ishibashi, J. Osuga, et al., Novel mutations in the microsomal triglyceride transfer protein gene causing abetalipoproteinemia, J. Lipid Res. 41 (2000) 1199-1204.

[50] E. Di Leo, S. Lancellotti, J.Y. Penacchioni, et al., Mutations in MTP gene in abetaand hypobeta-lipoproteinemia, Atherosclerosis 180 (2005) 311-318.

[51] M.W. Clarke, A.J. Hooper, H.A. Headlam, et al., Assessment of tocopherol metabolism and oxidative stress in familial hypobetalipoproteinemia, Clin. Chem. 52 (2006) 1339-1345.

[52] L. Chardon, A. Sassolas, B. Dingeon, et al., Identification of two novel mutations and long-term follow-up in abetalipoproteinemia: a report of four cases, Eur. J. Pediatr. 168 (2009) 983-989.

[53] N. Uslu, F. Gurakan, A. Yuce, et al., Abetalipoproteinemia in an infant with severe clinical phenotype and a novel mutation, Turk. J. Pediatr. 52 (2010) 73-77.

[54] M.B. Hammer, G. El Euch-Fayache, H. Nehdi, et al., Clinical features and molecular genetics of two Tunisian families with abetalipoproteinemia, J. Clin. Neurosci. 21 (2014) 311-315.

[55] E. Reboul, Vitamin E Intestinal Absorption: Regulation of Membrane Transport across the Enterocyte, IUBMB Life, 2018 PMID: 30308094. 